\title{
Transición entre sistemas financieros bancarios y bursátiles. Una aproximación mediante modelo de Swithing Markov ${ }^{1}$ \\ Transitions between banking and stock market financial systems. A Switching Markov model approach
}

DOI: https://doi.org/10.17981/econcuc.40.1.2019.08

Artículo de Investigación. Fecha de recepción: 18/02/2019 Fecha de aceptación: 15/04/2019

\author{
Christian Camilo Anzola Morales \\ Universidad Militar Nueva Granada. (Bogotá, Colombia) \\ camilo-anzolam@gmail.com \\ Paola Sophia Vargas Pachón \\ Universidad Militar Nueva Granada. (Bogotá, Colombia) \\ pao.vpn@gmail.com \\ Alberto Méndez Morales \\ Universidad Militar Nueva Granada. (Bogotá, Colombia) \\ edgard.mendez@unimilitar.edu.co
}

Para citar este artículo

Anzola, C., Vargas, P. y Méndez, A. (2019). Transición entre sistemas financieros bancarios y bursátiles. Una aproximación mediante modelo de Swithing Markov. Económicas CUC, 40(1). 123-144. DOI: http://doi.org/10.17981/econcuc.40.1.2019.08

\section{Resumen}

Los sistemas financieros se consideran como uno de los factores determinantes para el crecimiento económico; estos sistemas típicamente se reúnen en dos categorías, bancarios y bursátiles. La literatura referente evidencia a factores como el crédito interno al sector privado, las acciones negociadas (\% del PIB), el PIB per-cápita y la inflación, pueden ser indicadores de transición de un tipo de estado financiero a otro. Por consiguiente, el aporte principal del presente artículo es determinar la probabilidad de transición entre estados del sistema financiero bancario a uno bursátil y viceversa, presentes en países latinoamericanos emergentes como México, Colombia, Brasil y Chile (pertenecientes a la Organización para la Cooperación y el Desarrollo Económicos-OECD). Para realizar estos cálculos se utiliza una metodología Switching Markov de dos estados como herramienta de análisis para el periodo de estudio comprendido entre 2000-2016, con datos provenientes del Banco Mundial en el grupo de información financiera. Los resultados obtenidos reflejan como a través del tiempo, la mayoría de los países se mantienen en un solo estado, es decir, a pesar de las condiciones económicas internacionales no existe transición o cambios de sistema financiero. Se concluye que los tomadores de decisiones en estos países deberían proponer políticas que encausarán a dichos países por un uso más apropiado de los mercados bursátiles como una forma de promover la industria de alta tecnología y los mercados de diversificación de riesgo necesarios para soportar dichas iniciativa.

Palabras clave: Sistemas financieros; cadenas de Markov; OCDE; bancos; bolsas de valores

Codigo JEL: C22, E44, G15

\section{Abstract}

Financial systems are considered to be one of the determinants of economic growth; these systems typically fall into two categories, banking and stock market. The literature on factors such as domestic credit to the private sector, traded shares (\% of GDP), GDP per capita and inflation can be indicators of transition from one type of financial state to another. Therefore, the main contribution of this article is to determine the probability of transition between states of the banking financial system to a stock market and vice versa, present in emerging Latin American countries such as Mexico, Colombia, Brazil and Chile (belonging to the OECD). To perform these calculations, a two-state Switching Markov methodology is used as an analysis tool for the study period 2000-2016, with data from the World Bank in the financial information group. The results obtained reflect how over time, most countries remain in a single state, ie, despite international economic conditions there is no transition or changes in the financial system. It is concluded that decision makers in these countries should propose policies that will guide these countries for a more appropriate use of the stock markets as a way to promote the high-tech industry and the risk diversification markets necessary to support such initiatives.

Keywords: Financial systems; Markov chains; OECD; banks; stock exchanges.

\footnotetext{
${ }^{1}$ Este artículo es derivado del proyecto de investigación INV-ECO-2583, titulado: "Los sistemas financieros y la financiación de la actividad innovadora privada en países de la OCDE" de la Universidad Militar Nueva Granada.
} 


\section{INTRODUCCIÓN}

Los sistemas financieros, sus instrumentos, y el marco regulatorio que los componen, son factores decisivos para determinar el crecimiento económico de un país. Las características de cada uno y su enfoque, ya sea bancario o bursátil, permiten diseñar políticas económicas que potencialicen los diferentes sectores productivos, sirviendo a la vez como una herramienta de política monetaria que contribuye a prolongar ciclos económicos favorables (Luintel, Khan, Arestis \& Theodoriditis, 2008).

Adicionalmente, estos sistemas se presentan como escenarios en los cuales los gobiernos pueden desarrollar políticas enfocadas hacia los objetivos que permitan consolidar una estabilidad financiera congruente y con ello, generar crecimiento económico en el mediano y largo plazo (Rajan \& Zingales, 2003; Allen, Gu \& Kowalewski, 2017). Por un lado, un sistema financiero bancario es característico de países en dónde el desarrollo industrial y la producción de bienes es predominante. Este tipo de sistemas permiten una reducción del riesgo en las inversiones, en la medida que están sujetas al tipo de proyecto que se va financiar y su rentabilidad en el mediano y largo plazo (Stulz, 2000; Ergungor, 2004; Prochniak \& Wasiak, 2017). Por otro lado, el sistema financiero bursátil se ha desarrollado en aquellos países que han presentado cambios sustanciales en la generación de nuevas tecnologías. De la misma manera, en estos países, el sector de servicios y los proyectos de alta rentabilidad sobresalen, dado que cuentan con mejores herramientas financieras para la administración del riesgo, la difusión de información y la asignación de capital; todo esto generado por la diversificación de las fuentes de financiamiento hacia las empresas (Allen \& Gale, 2000; Ergungor, 2004; Allen, Bartiloro, Gu \& Kowalewski, 2018).

En complemento a lo anterior, Herger, Hodler \& Lobsiger (2008) define que la construcción y el diseño de un sistema financiero sofisticado, está estrechamente relacionado con la estabilidad política, económica y social de un país. Estos autores entienden como la heterogeneidad de contextos crea escenarios de transición de un estado bancario a bursátil o viceversa, lo cual puede afectar directamente la rentabilidad de las instituciones, los incentivos ofrecidos a la población para hacer uso de cada sistema y, sobre todo, el comportamiento que puede tener la política monetaria, debido a la restricción o expansión del crédito generado hacia el sector empresarial de manera directa (Levine, 1997; 2002). En este marco, las acciones que realiza un país para enfocarse por un sistema financiero u otro, buscan generar escenarios de crecimiento económico en los cuales se aumenta la demanda de crédito bancario, o en contraste, cuando se presenta una contracción, en el cual el crédito privado disminuye, incrementando la demanda de títulos bursátiles como una forma de financiamiento externo (Betancourt, Misas y Bonilla, 2011).

Teniendo presente lo anterior, se encuentra que en países en vía de desarrollo como México, Colombia y Chile, la transición entre los sistemas financieros (Bancario - Bursátil y viceversa) se ha facilitado en el tiempo gracias a las modificaciones de las políticas públicas, y a las condiciones socio-económicas, que han permitido un escenario de constante cambio ajustándose a las necesidades financieras de cada uno de estos países (Allen et al., 2018), sin embargo, estas 
transiciones parecen no afectar en profundidad el crecimiento económico de estos países que aun parecen rezagados frente a países desarrollados.

Por otro lado, existen características estructurales de cada economía que permiten determinar si existe un sistema financiero apropiado para un país, pero al tener en cuenta la relación de causalidad existente entre asignación de crédito, crecimiento y volatilidad, esta expectativa por determinar y fijar un sistema financiero pierde exactitud, dado que involucra la noción de sistema financiero como causa o consecuencia del crecimiento económico (Pussetto, 2008). Pese a esta disyuntiva, en el debate actual se ha coincidido, más allá de la estructura bancaria o bursátil, el punto clave es analizar la incidencia que tienen los costos de transacción e información asimétrica presentes en el mercado, y el rol que tienen los intermediarios financieros al convertirse en agentes supervisores de las asimetrías existentes en el mercado (Freixas \& Rochet, 2008).

Con los planteamientos preliminares, el aporte principal de este artículo es analizar la forma en que países como Colombia, Chile, Brasil o México tienen la posibilidad de generar una transición entre la forma actual de sistema financiero que enfrentan una diferente, que, aunque no necesariamente es mejor, si podría llevar a cambios estructurales de sus economías. De igual forma, se realiza una comparación con países con un sistema financiero más estable como Alemania y Japón, y que presentan una característica particular al considerarse como países con sistemas financieros bancarios; de igual manera, se incluye a Estados Unidos, categorizado como un país que presenta un sistema financiero bursátil fortalecido y a Reino Unido caracterizado por un sistema financiero mixto (Demirgüç-Kunt \& Levine, 2001; Lee,
2012) para comparar las transiciones de éstos, comparadas con las de los ya nombrados países de Latinoamérica.

La metodología usada en este documento se basa en el uso de cadenas de Márkov como herramienta de análisis del comportamiento de determinadas variables de la economía de los países seleccionados, y otros procesos estocásticos asociados al cambio de estado (sistema financiero bancario bursátil) a través del tiempo. El periodo de estudio se encuentra entre el año 2000 al 2016, periodo caracterizado especialmente por el acaecimiento de la crisis financiera sub-prime en los años 2008-2009. Para llevar a cabo este documento, se ha hecho uso de información pública del Banco Mundial en su dimensión de información financiera.

\section{REVISIÓN DE LITERATURA}

Calvo, Parejo, Rodríguez y Cuervo (2014) consideran los sistemas financieros como un conjunto de instituciones e intermediarios que logran captar y canalizar el exceso de efectivo y ahorro de los consumidores, enfocando las inversiones dentro de un mercado definido y dividido por unas estructuras bancarias y de mercado de capitales. Con esta premisa, estos sistemas comunmente cumplen con seis funciones fundamentales:

1. Proveer pagos para el intercambio de bienes y servicios,

2. agrupar fondos para las empresas,

3. transferir fondos económicos a través del tiempo hacia las diferentes regiones e industrias,

4. disminuir la incertidumbre y controlar el riesgo,

5. proveer información de precios para la toma de decisiones descentralizadas $\mathrm{y}$,

6. solucionar parcialmente los problemas de información asimétrica en los mercados (Merton, 1995). 
Partiendo de la anterior categorización, en la literatura se presenta una distinción entre los sistemas financieros existentes, bajo los cuales se muestra un extenso debate sobre cuál tipo de sistema es el más idóneo para incentivar el crecimiento económico, un sistema financiero bursátil o un sistema financiero bancario. Para lo cual Allen et al. (2017) y Demirgüç-Kunt \& Levine (2001) infieren que, la disparidad entre los sistemas se fundamenta en la divergencia existente en los procesos de evolución y diseño de cada uno, y es una respuesta regulatoria de los gobiernos a las dinámicas económicas, añadiendo que, su favorabilidad, se sustenta en la convergencia y similitud en las tasas de crecimiento económico de los países.

En este contexto, se puntualizan algunas características teóricas de los sistemas bancarios y bursátiles que permiten analizar su disparidad, y conllevan a fortalecer la interpretación del propósito planteado en este documento. En primer lugar, los países que son intensivos en agricultura e industria, tienden a exhibir un sistema bancario más sólido, lo cual permitiría un aumento de la liquidez para los proyectos realizados por este tipo de industrias. Segundo, los países que desarrollan y se especializan en el sector servicios, incentivan en mayor medida la rentabilidad y el fortalecimiento de la participación del desarrollo innovativo en las industrias, se caracterizan por presentar un sistema bursátil más desarrollado (Rajan \& Zingales, 2003; Ergungor, 2004; Allen et al., 2018)

En concordancia, en lo referente a los sistemas bancarios, se puede decir que estos son característicos de economías pioneras e intensivas en el desarrollo industrial, mediante recursos proporcio- nados por conglomerados bancarios que financian por etapas de evolución y actividades de producción que implican una identificación de proyectos con alta rentabilidad (Levine, Beck \& Maksimovic, 2000; Stulz, 2000). Gerschenkron (1962) y Rajan \& Zingales (1998) fundamentan este sistema en estrechas relaciones a largo plazo con la industria y cuentan con una ventaja comparativa frente al sector bursátil ya que cuentan con mayor disponibilidad de información financiera de las industrias y cuentan con una red estructurada de normas, procedimientos, mecanismos y soportes institucionales que preservan su estabilidad. Algunos de los países sobresalientes en este sistema son Francia, Alemania y Japón.

Por otro lado, el sistema financiero bursátil es característico de países como Estados Unidos y Canadá, siendo, según Russ \& Valderrama (2012) y Zogning (2017), una estructura que depende directamente de las dinámicas del mercado financiero externo. En estos países, se destacan las oportunidades de los inversionistas de transar instrumentos financieros como acciones, bonos, commoditties y derivados. De igual manera, estos sistemas se consideran una respuesta a los cambios en industrias innovadoras, que a su vez, permiten mejoras significativas en el sistema legal dado que se fortalece la protección del inversionista sobre sus derechos de propiedad (Prochniak \& Wasiak, 2017), y contribuye a la creación de empresas que diversifican el riesgo (Levine \& Zervos, 1999). Considerando lo expuesto, se observa que implementar un tipo de sistema financiero no es excluyente de disponer de algunas características del otro, esto implicaría que en algunos países se presente una combinación de los dos sistemas (Aghion, Howitt \& Mayer- 
Foulkes, 2005) como es el caso del Reino Unido.

En este sentido, Terceño y Guercio (2011) a través del desarrollo de un modelo básico de correlaciones, y Allen et al. (2018), mediante un modelo de mínimos cuadrados ordinarios (OLS) y el método GMM en dos pasos, convergen en el planteamiento de variables como activos bancarios, circulación de bonos públicos y privados, crédito público y privado, y capitalización bursátil; por medio de estas variables concluyen que, aquellos países que son intensivos en activos tangibles presentan la tendencia a tener un sistema financiero bancario extenso, generando un crecimiento industrial en el largo plazo, bajo la condición de que la estructura económica se siga desenvolviendo dentro de la producción de bienes. Por el contrario, aquellos países que se encuentran definidos por la producción de servicios o intangibles, se caracterizan por industrias que se financian a través de bonos o acciones, característicos de sistemas bursátiles. Esto último tiene sentido en la medida en que los bancos, al evaluar la concesión de un crédito, exigirán activos de respaldo a las compañías, activos que generalmente son del tipo tangible, con posibilidades de reventa y, por tanto, con alta liquidez. De igual forma, los sistemas financieros bursátiles se presentan en mercados con alto nivel de activos intangibles ya que la comercialización de estos conlleva a un alto riesgo de recuperación de la inversión, lo cual eleva el riesgo, pero a su vez la rentabilidad esperada de las empresas.

De igual forma Bruno, De Bonis \& Silvestrini (2012), mediante la utilización de un modelo de mínimos cuadrados ordinarios (OLS) y con la inclusión de va- riables como depósitos, valores de deuda, productos de seguros y acciones, realizan un estudio para los países pertenecientes al G7 y la OCDE, abarcando el periodo 1980-2005. Los autores encuentran que, para el caso de los Estados Unidos, se evidencia una contracción en el comportamiento de la banca y los depósitos postales a raíz de la fuerte presión ejercida por los mercados bursátiles en el desarrollo de su competencia. Sin embargo, para el caso de Alemania y Japón, la presencia de los depósitos sigue siendo significativa respecto al mercado accionario, predominando la banca. Adicionalmente, para los países de la OCDE se evidencia convergencia entre los sistemas financieros delimitados no solamente para las acciones y productos de la industria de seguros, sino también para los depósitos y títulos de deuda, generando una relación estrecha al subdesarrollo financiero en la década de los 60 y en la propagación de los instrumentos en los años posteriores. Este tipo de sistemas financieros descritos por los autores, es posible observarlo a su vez en países en vía de desarrollo con una alta concentración bancaria y con bajos retornos de inversión en el corto y largo plazo.

En relación a los modelos econométricos utilizados, existe literatura alterna como Kaufmann \& Valderrama (2008), que evalúan los sistemas financieros de Alemania y el Reino Unido entre 1980 a 2006, mediante la metodología de cadenas de Markov y su derivación del Vector Autorregresivo Switching Markov (VAR), delimitado como un cambio de estado que presenta una variable. Los autores encuentran que, los préstamos a corporaciones no financieras están impulsados por la demanda de estos países, y que las empresas de los mismos, no presentan 
una fuerte restricción crediticia. De esta forma, los resultados reflejan que, por un lado, Alemania se define por un sistema financiero fundamentado en bancos, en la medida que los choques en los préstamos generan un efecto positivo sobre el crédito privado cuando el crecimiento se sostiene inferior a la media. Por otro lado, en el caso del Reino Unido, las crisis crediticias han generado un efecto positivo sobre la inversión, aumentando el financiamiento de la industria a través del mercado de valores articulado a su vez, de un sistema bancario que se ha mezclado con el mercado bancario en este país. En otras palabras, el sistema financiero del Reino Unido es de tipo mixto.

Teniendo en cuenta lo anterior, Beck \& Levine (2002) y Levine (2002), mediante una evaluación empírica de la estructura financiera y por medio del uso de datos panel para 42 países y 36 industrias concluyen que, aunque no es relevante analizar a los países por tipo de sistemas financieros para explicar su crecimiento económico, el hecho de que los países tengan sistemas financieros eficientes, y los inversionistas tengan la posibilidad de acceder a financiamiento externo, puede crear un escenario de crecimiento de las industrias, en la medida que se crean nuevas organizaciones, empleo e incentivos para el crecimiento económico.

Al tener presente los aspectos anteriormente referenciados, el aporte principal de la presente investigación a la literatura es, determinar la probabilidad de que los países estudiados generen una transición entre sistemas financieros, haciendo uso del Vector Autorregresivo Switching Markov y la caracterización de cada uno de los países latinoamericanos pertenecientes a la OECD y comparando a su vez éstos con países como Alemania, para el caso del sistema financiero bancario y Estados Unidos para el sistema financiero bursátil. Adicionalmente, se cuenta con Japón y Reino Unido, destacándose por poseer un sistema financiero mixto.

\section{Desarrollo}

Para estudiar el posible cambio de estado de un sistema financiero a otro en los países que conforman los dos bloques económicos enunciados, se ha usado el Banco Mundial como fuente de datos. En primer lugar, se ha utilizado la información de transformación financiera entre los periodos de 2000-2016, extrayendo la data de la variable "Acciones negociadas (\% del PIB) (Acc_PIB)", la cual es catalogada como variable dependiente y permite visualizar el nivel de bursatilización de activos en el sistema financiero del promedio de todos los países de estudio. Reflejada en la figura 1 , variable con cambios sustanciales en la primera década del periodo estudiado, en la medida que el promedio de las acciones negociadas disminuye hasta un $39,33 \%$ para el año 2002 ; y posteriormente, se evidencia un repunte llegando a un valor de $94,59 \%$ para el año 2007 . Con ello infiriendo que, hasta ese momento se presentaba un mejoramiento del sector bursátil para todos los países en el periodo de tiempo estudiado. Sin embargo, en los años siguientes, la dinámica presenta una tendencia negativa fundamentada en dos situaciones específicas: la primera, la crisis financiera sub-prime que repercutió en los mercados internacionales en el año 2008 y la segunda, el deterioro generalizado del ambiente macroeconómico que llevo a la disminución de la actividad económica y al aumento de las tasas de interés. 


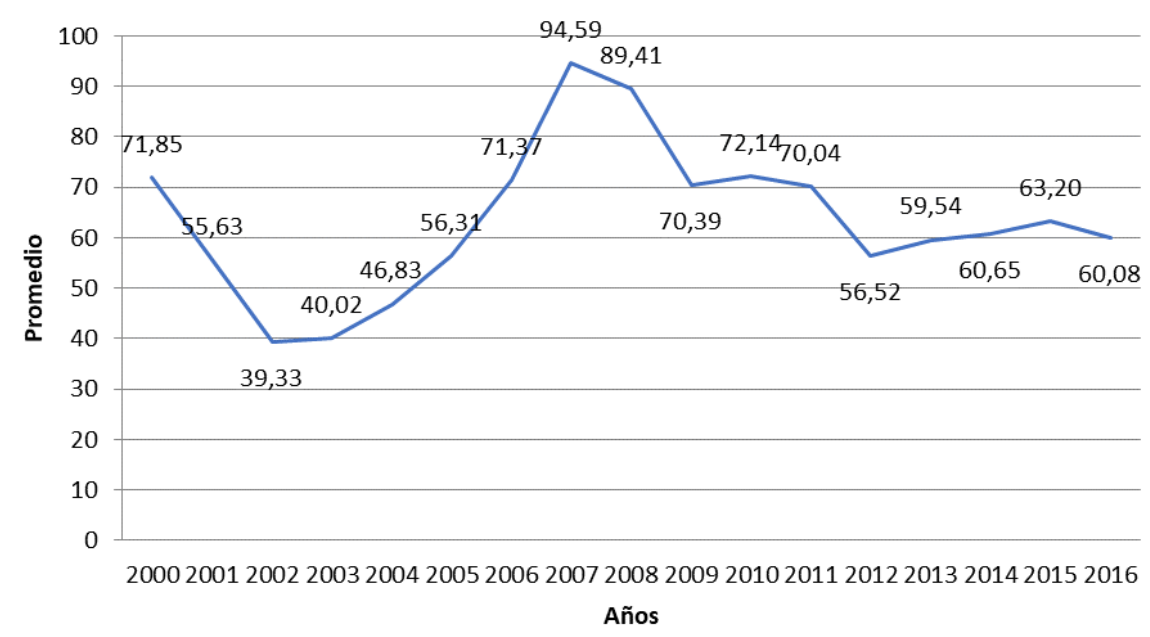

Figura 1. Promedio evolución de Acciones negociadas - (\% del PIB). Total de países. Fuente: Elaboración propia a partir de Banco Mundial.

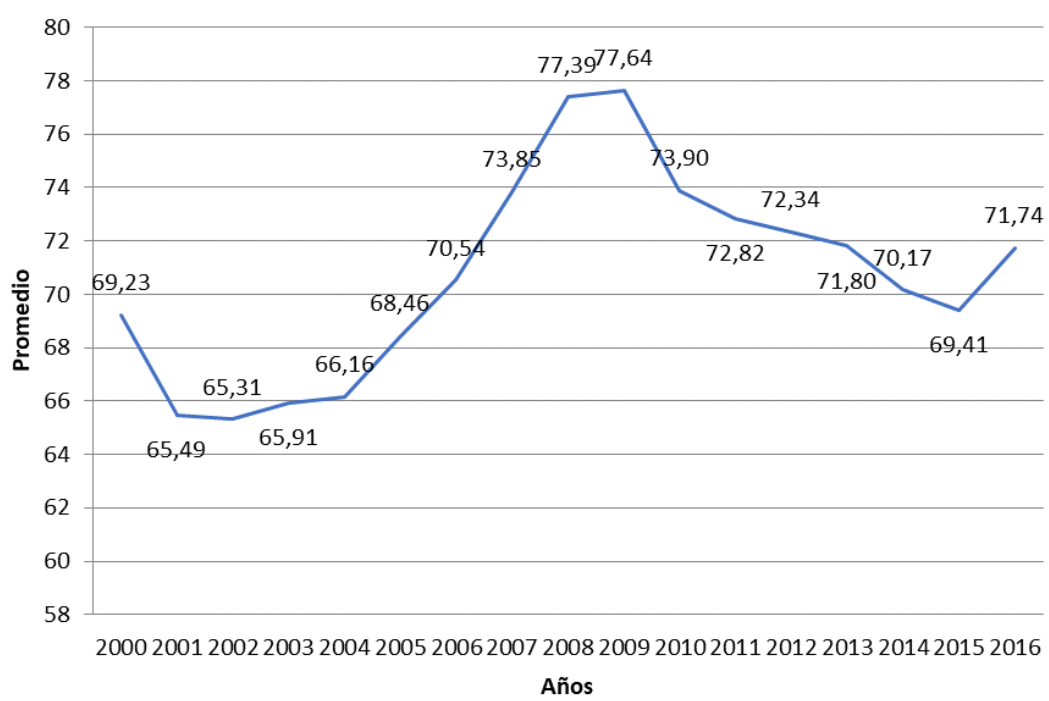

Figura 2. Promedio Crédito interno al sector privado por los bancos (\% del PIB).

Total de países.

Fuente: Elaboración propia a partir de Čihák, Demirgüç-Kunt, Feyen \& Levine, 2012.

Dentro del conjunto de variables independientes, se toma inicialmente la evolución de la variable "Crédito interno al sector privado por los bancos (\% del PIB) (Cred_PIB)" como referente de expectativa generada en la crisis financiera del año 2008 y sus consecuencias sobre el desarrollo financiero de los países de estudio. Presentada en la figura 2 , esta variable refleja que, para los primeros 8 años (hasta la crisis del año 2008), existía una dinámica creciente pasando del $69.23 \%$ al $77.39 \%$ de los créditos privados que se han otorgado al sector privado en todos los países objeto de estudio como porcentaje del PIB. Posterior a esta fecha, se percibe una tendencia negativa, evidenciando la fuerte disminución que ha tenido la demanda de este tipo de instrumentos financieros. 
De igual forma, para tener un bosquejo de la estabilidad de las economías estudiadas, se incluyen variables independientes como el "PIB per cápita (PIB_perc)" (figura 3) y la "inflación (Inflac)" (figura 4). La selección de estas variables, permiten entender su validez y descartar empíricamente problemas de variables omitidas. Por un lado, la figura 3 manifiesta el comportamiento promedio del PIB per-cápita para todos los países estudiados. Reflejándose una fuerte variación a través del tiempo, especialmente en la crisis financiera de este siglo, que llevó a una fuerte contracción de la producción nacional en términos generales y una fuerte recuperación en años posteriores, y a su vez, infiriere una tendencia negativa hasta el 2016.

Por otro lado, el comportamiento promedio de la inflación en la figura 4 evidencia a través del tiempo que, a pesar de que existen algunos picos positivos o de crecimiento la tendencia de la inflación es una variación negativa, conllevando a un control monetario consistente a las condiciones externas en cada uno de los países, evitando de esta forma fuertes caídas en la producción nacional.

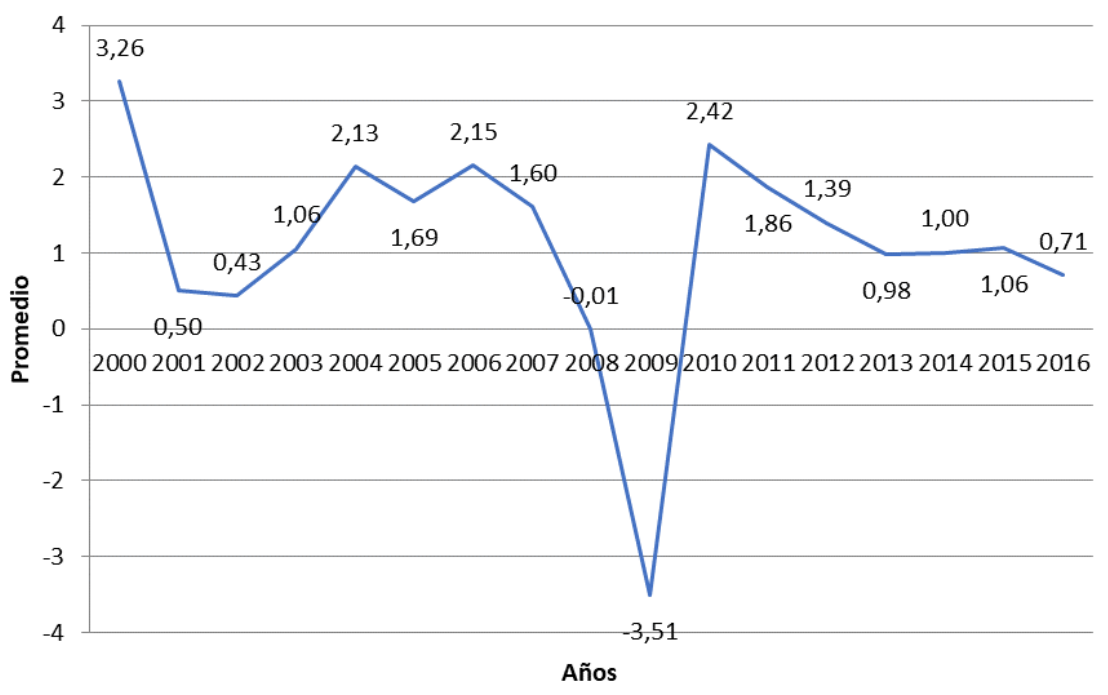

Figura 3. Promedio Crecimiento porcentual del PIB per-cápita - Total de países. Fuente: Elaboración propia a partir de Čihák, Demirgüç-Kunt, Feyen \& Levine, 2012.

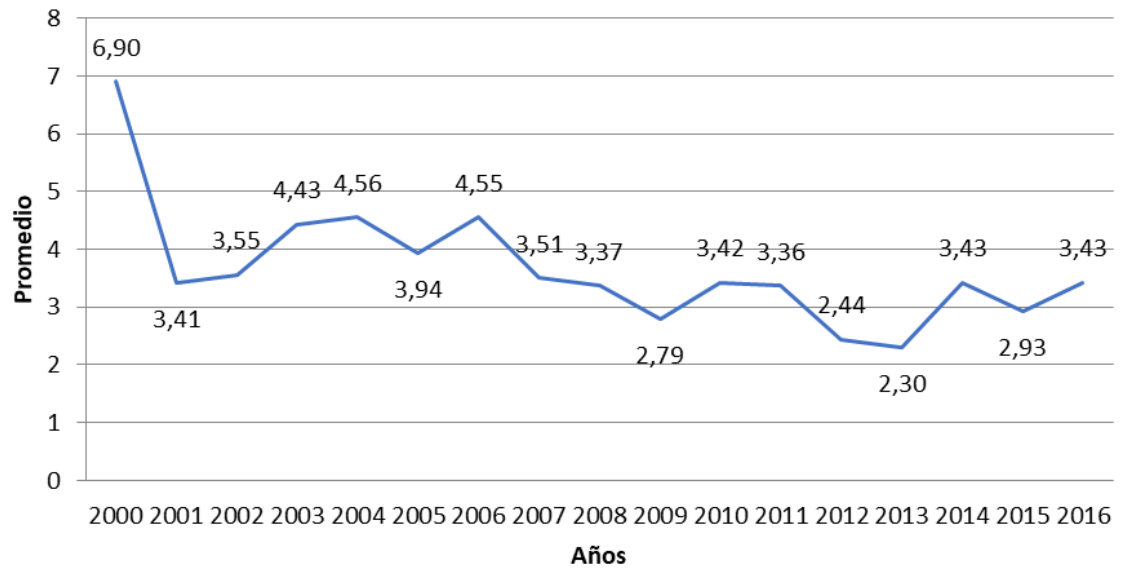

Figura 4. Promedio inflación. Total de países.

Fuente: Elaboración propia a partir de Čihák, Demirgüç-Kunt, Feyen \& Levine, 2012. 
En complemento al análisis de comportamiento anterior, en la tabla 1 se detalla la distribución porcentual de los países analizados en este artículo, con una muestra de 17 observaciones para cada uno, y para la variable acciones negociadas (\% del PIB). Se evidencia la mayor cantidad de acciones negociadas desde el año 2000 al 2016 es de USA con el $223.05 \%$ del PIB, y en menor proporción, México con una media del $8.26 \%$ del PIB.
En este mismo sentido, la tabla 2 enfocada en el crédito interno al sector privado (\% del PIB) refleja como el Reino Unido es el país con la mayor cantidad de créditos asignados al sector privado con una media de $150.98 \%$ durante los 16 años analizados. De forma similar, Japón presenta un promedio de créditos que rodean los $105.60 \%$. Teniendo presente que países como Brasil y Alemania, solamente cuentan con 16 observaciones.

TABLA 1

Estadísticas descriptivas por país - Acciones negociadas (\% del PIB)

\begin{tabular}{cccccc}
\hline País & $\mathrm{N}$ & Media & Desviación estándar & Min. & Máx. \\
\hline México & 17 & 8.26 & 2.65 & 3.24 & 12.97 \\
Colombia & 17 & 5.25 & 3.14 & 0.80 & 9.61 \\
Brasil & 17 & 26.29 & 11.43 & 7.75 & 46.20 \\
Chile & 17 & 14.44 & 6.76 & 3.82 & 26.51 \\
Alemania & 17 & 53.94 & 24.24 & 32.32 & 111.65 \\
Japón & 17 & 85.96 & 33.28 & 39.55 & 145.86 \\
Reino Unido & 15 & 93.83 & 26.06 & 59.24 & 131.62 \\
Estados Unidos & 17 & 223.05 & 50.14 & 139.39 & 320.99 \\
\hline
\end{tabular}

Fuente: Elaboración propia a partir de Čihák, Demirgüç-Kunt, Feyen \& Levine, 2012.

TABLA 2

Estadísticas descriptivas por país - Crédito interno al sector privado por los bancos (\% del PIB)

\begin{tabular}{lccccc}
\hline \multicolumn{1}{c}{ País } & $\mathrm{N}$ & Media & Desviación estándar & Min. & Máx. \\
\hline México & 17 & 17.93 & 4.22 & 12.12 & 26.69 \\
Colombia & 17 & 28.34 & 5.67 & 20.76 & 36.37 \\
Brasil & 16 & 46.85 & 15.04 & 27.69 & 66.78 \\
Chile & 17 & 69.30 & 6.67 & 61.61 & 81.14 \\
Alemania & 16 & 94.24 & 12.56 & 77.07 & 112.04 \\
Japón & 17 & 105.60 & 20.80 & 93.38 & 184.71 \\
Reino Unido & 17 & 150.98 & 25.36 & 115.16 & 194.39 \\
Estados Unidos & 17 & 52.49 & 3.43 & 48.81 & 59.75 \\
\hline
\end{tabular}

Brasil y Alemania solamente cuentan con 16 observaciones.

Fuente: Elaboración propia a partir de Čihák, Demirgüç-Kunt, Feyen \& Levine, 2012. 
La tabla 3 por su parte, hace referencia a la distribución correspondiente a la variación del PIB per-cápita de los países. En este sentido, se puede observar que Chile es el país que lidera el crecimiento del PIB durante los 16 años de estudio, con una variación promedio de $3.01 \%$, seguido por Alemania con un $1.30 \%$ y el Reino Unido con una media de $1.19 \%$.

En el caso de la tabla 4, se analiza la variación de la inflación presente en los países latinoamericanos con el mayor índice de inflación frente a los países desarrollados. Particularmente, Brasil representa este grupo con una media de $8.16 \%$ y Japón con un promedio de $-0.68 \%$.

La figura 5 describe la evolución del crédito bancario al sector privado como porcentaje del PIB, variable que contribuye a entender como, por país se ha impulsado una política bancaria o bursátil del sistema financiero.

TABLA 3

Estadísticas descriptivas por país - Variación promedio del PIB per-cápita 2000-2016

\begin{tabular}{cccccc}
\hline País & $\mathrm{N}$ & Media & Desviación estándar & Min. & Máx. \\
\hline México & 17 & 0.93 & 2.47 & -6.22 & 3.83 \\
Colombia & 16 & 0.03 & 0.02 & 0.00 & 0.06 \\
Brasil & 16 & 0.01 & 0.03 & -0.05 & 0.06 \\
Chile & 17 & 3.01 & 2.13 & -2.54 & 6.04 \\
Alemania & 17 & 1.30 & 2.42 & -5.38 & 5.60 \\
Japón & 17 & 0.89 & 2.02 & -5.41 & 4.17 \\
Reino Unido & 17 & 1.19 & 1.87 & -4.91 & 3.29 \\
Estados Unidos & 17 & 1.07 & 1.59 & -3.62 & 2.94 \\
\hline
\end{tabular}

Colombia y Brasil solamente cuentan con 16 observaciones.

Fuente: Elaboración propia a partir de Čihák, Demirgüç-Kunt, Feyen \& Levine, 2012.

TABLA 4

Estadisticas descriptivas por país - Inflación

\begin{tabular}{cccccc}
\hline País & $\mathrm{N}$ & Media & Desviación estándar & Min & Max \\
\hline México & 17 & 5.26 & 2.06 & 1.76 & 10.82 \\
Colombia & 17 & 6.57 & 6.75 & 1.99 & 31.76 \\
Brasil & 17 & 8.16 & 1.78 & 5.89 & 14.09 \\
Chile & 17 & 4.87 & 2.99 & 1.13 & 12.16 \\
Alemania & 17 & 1.19 & 0.64 & 0.30 & 2.01 \\
Japón & 17 & -0.68 & 1.10 & -1.90 & 2.04 \\
Reino Unido & 17 & 1.99 & 0.68 & 0.46 & 3.03 \\
Estados Unidos & 17 & 1.96 & 0.68 & 0.76 & 3.22 \\
\hline
\end{tabular}

Fuente: Elaboración propia a partir de Čihák, Demirgüç-Kunt, Feyen \& Levine, 2012. 


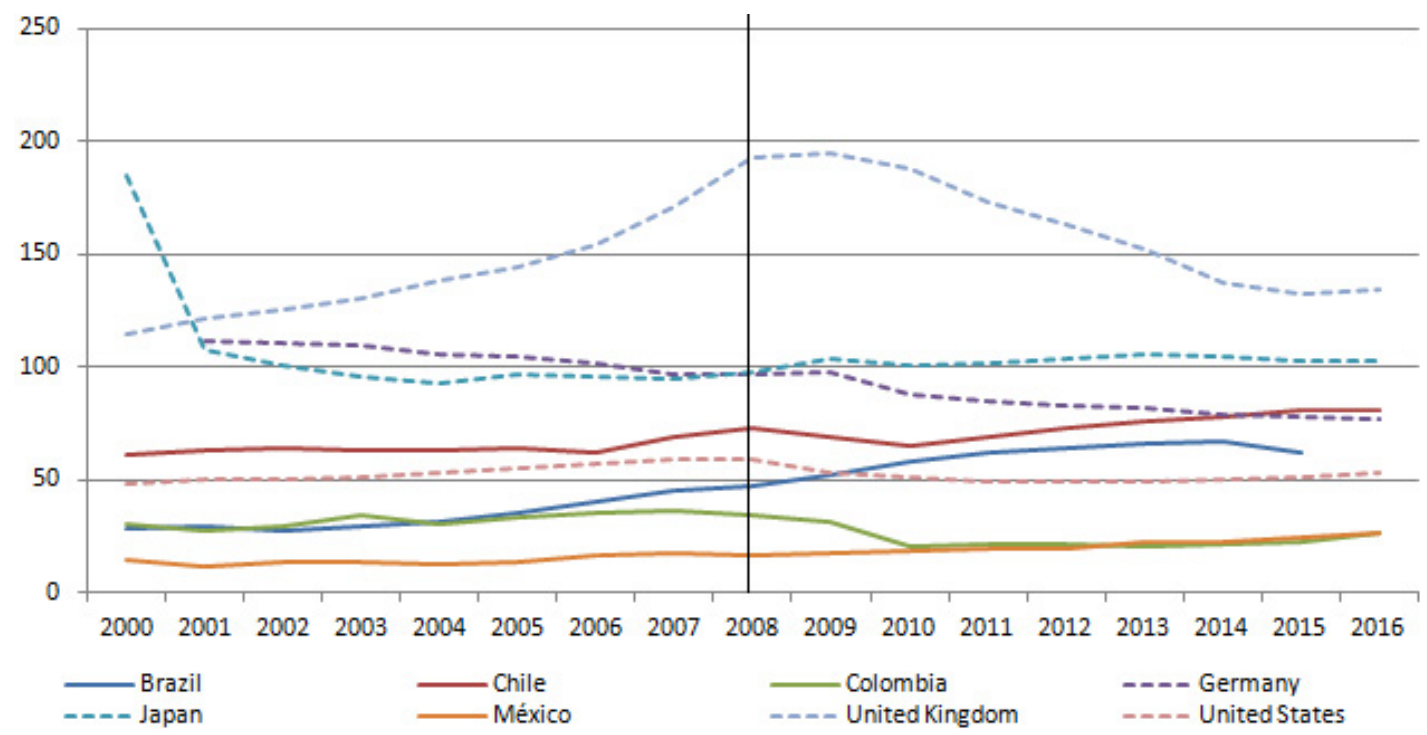

Figura 5. Crédito bancario al sector privado como porcentaje del PIB - Total países. Fuente: Elaboración propia a partir del International Monetary Fund, International Financial Statistics and data files, and World Bank and OECD GDP estimates (2018).

Los datos utilizados en la elaboración del modelo permiten construir dos estados con una tasa moderada de variación, al contar con poca incertidumbre entre momentos, y genera un escenario ideal que permite asumir un modelo sin problemas de heteroscedasticidad. Teniendo en cuenta los dos estados o regímenes, se determina por medio de la media y la varianza una diferenciación en la media representada por etapas expansivas $\left(u_{1} \geq 0\right)$ de la variable dependiente y la varianza de las etapas contractivas $\left(u^{2}<0\right)$. La figura 6 muestra el proceso de decisión del modelo para determinar los estados en que se ubica.

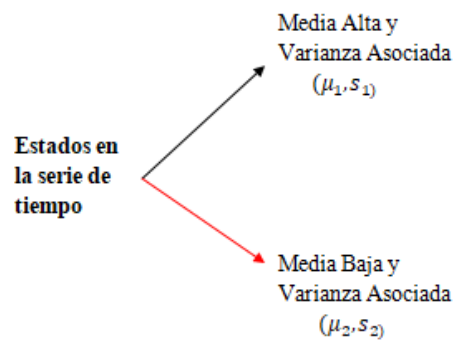

Figura 6. Restricción de estados según varianza de los datos.

Fuente: (Goutte, 2014).

\section{Estrategia empírica}

Inicialmente, es necesario referenciar que las cadenas de Markov se consideran como un proceso estocástico y paramétrico, en el cual se refleja el cambio del estado en una variable influenciada por sus eventos pasados, teniendo en cuenta que cada transición o cambio, es realizado en tiempo discreto (López y Joa, 2001; Dong, Hong \& Myung, 2018), permitiendo estudiar un efecto lineal de las series de tiempo. Con lo anterior, se estima un modelo de regresión dinámica Switching Markov de dos estados. Con éste, se busca identificar la probabilidad de transición de un tipo de sistema financiero a otro, los cuales están categorizados como:

1. Sistema financiero basado en mercados bancarios.

2. Sistema financiero basado en mercados de capitales.

La metodología aplicada a nuestra estrategia empírica, parte de la idea de que existen efectos asimétricos en cada tipo de sistema, para lo cual, el modelo Swichting 
Markov incluye un vector autorregresivo (M-S), que permite determinar los coeficientes de cambio de régimen basado en bancos o basado en mercados de capitales. En el presente caso, se especifican dos tipos de modelos 1 y 2 , de forma separada de la siguiente manera:

$$
\begin{aligned}
& \text { Estado } 1=Y_{t}=\mu_{1}+\theta y(t-1)+\varepsilon t \\
& \text { Estado } 2=Y_{t}=\mu_{2}+\theta y(t-1)+\varepsilon t
\end{aligned}
$$

En este, $S_{t}$ es la variable latente de primer orden $\left\{S_{t}\right\}_{t=1}^{T}, S t=1$ es un sistema financiero bancario y $S t=2$ un sistema financiero bursátil, $\theta$ representa los parámetros autorregresivos que componen la función de probabilidad de estados y finalmente $\varepsilon$, es un error de ruido blanco con varianza $\theta^{2}$. Estas variables, de acuerdo al estado o régimen en el que se encuentren (1 o 2), siguen la siguiente distribución:

$$
\begin{aligned}
& \text { Estado 1: } \mathrm{N}\left(\mu_{1}, \sigma_{1}{ }^{2}\right) \\
& \text { Estado 2: } \mathrm{N}\left(\mu_{2}, \sigma_{2}{ }^{2}\right)
\end{aligned}
$$

Es así que el comportamiento de la variable dependiente de $Y_{t}$ es independiente sobre $S_{t}$ de forma que:

$$
y_{t} / s_{t} \approx \mathrm{N}\left(\mu_{i}, \sigma_{i}^{2}\right)
$$

Con una función de densidad $Y_{t}$, condicional a $S_{t}$ :

$$
f\left(y_{t} / S_{t}\right)=\frac{1}{\sqrt{2 \Pi \sigma S_{t}}} \exp \left(\frac{-\left(y_{t}-S_{t}\right)^{2}}{2 \sigma^{2} S t}\right)
$$

La variable latente del cambio de régimen $S_{t}$ se caracteriza de acuerdo a la matriz de probabilidad $P_{t}(7)$.
En esta matriz $p_{i j}$ es la probabilidad de pasar del estado $i$ en el periodo $t_{-1}$ al estado $j$ en el periodo $t$, y $F$ es una función de distribución acumulativa normal. Los elementos del vector $(k \times 1) x_{t-1}$ son las variables que afectan la probabilidad de transición de $\beta$ y son parámetros estados dependientes y que deberán ser estimados.

Para el análisis, inicialmente se declara una serie de tiempo incluyendo valores por un periodo del 2000 a 2016 . En este periodo se determinan las siguientes variables: Total de acciones negocias como parte del PIB, crédito bancario al sector privado como porcentaje del PIB, variación de PIB per cápita e inflación.

Estas variables, además del argumento coyuntural que explica el impacto de las mismas sobre el cambio de régimen, contribuyen a conformar un modelo con una función de probabilidad más ajustada. Por la naturaleza de la hipótesis, en la que los resultados se ajustan a la evolución de variables observadas, la selección del modelo final se enmarcó en los criterios que permitieron hacer uso de la función de Log-likehood (máxima verosimilitud), es decir los criterios de información ${ }^{2}$ de Akaike (AIC) y el criterio de información Bayesiano (BIC) por sus siglas en inglés. Estos criterios fueron los más validos entre los modelos planteados ex ante de la selección del modelo final. Las estimaciones para el modelo final en comparación al modelo más cercano fueron menores en todos los casos y se observan en la tabla (5).

\section{Momento t}

Estado 1

Momento t-1 Estado $1 \quad p_{11}^{t}=F\left(x_{t-1} \beta_{-1}\right)$

Estado $2 \quad p_{21}^{t}=1-F\left(x_{t-1} \beta_{-2}\right)$
Estado 2

$$
\begin{gathered}
p_{12}^{t}=1-F\left(x_{t-1} \beta_{-1}\right) \\
p_{22}^{t}=F\left(x_{t-1} \beta_{-2}\right)
\end{gathered}
$$

${ }^{2}$ Cuando se tiene una serie de modelo M1 y M2 con parámetros K1 y K2 respectivamente, una metodología para compararlos corresponde a la función de máxima verosimilitud (likelihood). Esta permite seleccionar el modelo que cumple con un mejor ajuste de los datos pero que no penaliza su complejidad. Los criterios AIC y BIC permiten identificar la mejor bondad de ajuste y la probabilidad relativa de escogencia del mejor modelo. En ambos casos, los modelos que presentan mejor valor en AIC y BIC son los mejores. 
TABLA 5

Resultados de evaluación de criterios - Ajuste al modelo

\begin{tabular}{ccccc}
\hline \multicolumn{5}{c}{ Criterio de selección } \\
\hline País & AIC Modelo Final & AIC Modelo 1 & BIC Modelo Final & BIC Modelo 1 \\
México & 4.42 & 6.01 & 4.86 & 6.25 \\
Colombia & 5.16 & 6.77 & 5.59 & 7.21 \\
Brasil & 7.21 & 8.25 & 7.53 & 8.58 \\
Chile & 5.99 & 9.74 & 6.44 & 9.99 \\
Alemania & 8.31 & 9.89 & 8.74 & 10.23 \\
Japón & 9.73 & 10.68 & 9.97 & 11.02 \\
Reino Unido & 8.77 & 9.00 & 9.20 & 9.25 \\
Estados Unidos & 8.77 & 11.02 & 9.19 & 11.26 \\
\hline
\end{tabular}

Fuente: Elaboración propia a partir de Čihák, Demirgüç-Kunt, Feyen \& Levine, 2012.

Estado 1: Acc_neg_\% $P I B_{t}=\mu_{1}+\theta_{1} c r e \_b a n \_\% P I B_{t}+\theta_{2} \Delta P I B \_p c_{t}+\theta_{3} I n f_{t}+\varepsilon t$
Estado 2: Acc_neg_\%PIB ${ }_{t-1}=\mu_{2}+\theta_{1} c r e \_b a n \% P I B_{t-1}+\theta_{2} \Delta P I B \_p c_{t-1}+\theta_{3} I n f_{t-1}+\varepsilon_{t}$

De esta manera, se construye un Markov-Switching Dynamic Regression (MSDR) de 2 estados con selección de variables que se enmarcan en una función de probabilidad más ajustada en donde los valores esperados de la variable dependiente no se rigen por un proceso estocástico no observable (8)

El interés de este planteamiento parte en determinar cómo surgen en los países de estudio las transiciones entre estados, lo cual se ha hecho evidente por la significancia de las variables independientes (1. crédito interno bancario al sector privado como porcentaje del PIB 2. variación del PIB per cápita y iii. inflación) y ha logrado asimilar los impactos entre variables. Sin embargo, la selección de un modelo de MSDR permite entender también las condiciones que permiten estar en un estado o no, relacionando la hipótesis planteada en este documento y la evolución de los ciclos económicos de cada país. Es así que la definición de los dos estados establecidos se basa en el régimen anterior al año 2008 y al establecido después del mismo año.

El documento no trata empíricamente el efecto per se de la crisis financiera sobre los regímenes establecidos para desarrollar el modelo, pero la evidencia y el análisis descriptivo si denota una clara variación en las variables de interés a partir de ese evento.

\section{Resultados}

En esta sección se presentan los resultados de referencia; estos surgen de las estimaciones realizadas con el MSDR y se encuentran en la tabla 7, en complemento con las tablas de resultados (anexos 8-13). El modelo es consistente en varianza, estadísticamente significativo a un nivel del $5 \%$ para la mayoría de las variables en los 
dos estados y en gran parte de los países. La intuición del análisis parte del comportamiento de la variable dependiente observada en la figura 5 y cómo la misma define el estado bancario, mixto o bursátil del que hace parte cada país, además de la probabilidad determinada en las estimaciones de las columnas (7) y (8) las cuales presentan el índice de probabilidad de permanencia y persistencia de cada país en cada uno de los estados. Esta persistencia explica la probabilidad de permanencia en un estado dado un cambio, mas no el cambio de estado per se. Las columnas (1) y (4) muestran la relación de significancia del nivel de crédito bancario al sector privado como porcentaje del PIB. Las columnas (2) y (5) muestran la significancia de PIB per cápita sobre la variable dependiente al igual que las columnas (3) y (6) que consideran la significancia del factor inflacionario sobre la misma. Adicionalmente, se observa la tabla 6 , en la cual se identifica de acuerdo a la significancia de las variables entre los estados 1 y 2 la persistencia de permanencia en un régimen $u$ otro. Estos comportamientos y resultados por país, se describen en las siguientes tablas (6 y 7$)$ :

TABLA 6

\section{Persistencia entre estados por país}

\begin{tabular}{ccc}
\hline País & Estado (1) & Estado (2) \\
\hline México & Bancario & Bancario \\
Colombia & Bancario & Bancario \\
Brasil & Mixto & Bancario \\
Chile & Bancario & Bancario \\
Alemania & Bancario & Bancario \\
Japón & Mixto & Mixto \\
Reino Unido & Bancario & Mixto \\
Estados Unidos & Bursátil & Bursátil \\
\hline
\end{tabular}

Fuente: Elaboración propia.

TABLA 7

Estimaciones de referencia - Resultados entre estados del MSDR

\begin{tabular}{ccccccccc}
\hline \multicolumn{7}{c}{ Estado 1 (P-value) } & \multicolumn{7}{c}{ Estado 2 (P-value) } \\
\hline País & Cred_ & $\begin{array}{c}\text { PIB_ } \\
\text { perc }\end{array}$ & Inflac & $\begin{array}{c}\text { Cred_ } \\
\text { PIB }\end{array}$ & $\begin{array}{c}\text { PIB_ } \\
\text { perc }\end{array}$ & Inflac & P (Estado 1) & P (Estado 2) \\
México & $0.00^{* *}$ & $0.00^{* *}$ & $0.016^{*}$ & $0.00^{* *}$ & 0.30 & $0.11^{*}$ & 0.86 & 0.57 \\
Colombia & 0.86 & 0.73 & 0.80 & $0.00^{* *}$ & $0.08^{*}$ & 0.70 & 0.90 & 0.94 \\
Brasil & $0.00^{* *}$ & $0.075^{*}$ & $\mathrm{ND}$ & $0.00^{* *}$ & 0.81 & $\mathrm{ND}$ & 0.92 & 0.67 \\
Chile & $0.16^{*}$ & 0.78 & $0.00^{* *}$ & $0.00^{* *}$ & 0.71 & $0.00^{* *}$ & 0.92 & 0.82 \\
Alemania & $0.00^{* *}$ & $0.01^{*}$ & 0.56 & $0.00^{* *}$ & $0.00^{* *}$ & $0.00^{* *}$ & 0.92 & 0.61 \\
Japón & $\mathrm{ND}$ & $\mathrm{ND}$ & $\mathrm{ND}$ & $\mathrm{ND}$ & $\mathrm{ND}$ & $\mathrm{ND}$ & 0.83 & 0.79 \\
$\begin{array}{c}\text { Reino } \\
\text { Unido }\end{array}$ & $0.00^{* *}$ & 0.44 & $0.12^{*}$ & $0.00^{* *}$ & $0.00^{* *}$ & $0.00^{* *}$ & 0.68 & 0.59 \\
$\begin{array}{c}\text { Estados } \\
\text { Unidos }\end{array}$ & $\mathrm{ND}$ & $\mathrm{ND}$ & $\mathrm{ND}$ & $\mathrm{ND}$ & $\mathrm{ND}$ & $\mathrm{ND}$ & 0.71 & 0.91 \\
\hline
\end{tabular}

Niveles de significancia * $0.11^{* * *} 0.05^{* * *} 0.01$

Fuente: Elaboración propia a partir de Čihák, Demirgüç-Kunt, Feyen \& Levine, 2012. 
Los resultados de la columna 7 indican que para el bloque de países latinoamericanos se tiene un alto nivel de probabilidad de permanencia y persistencia en un primer estado de sistema financiero y como sugiere la figura 5 , se tiene una marcada tendencia a ser bancario. Se evidencia para Colombia y Chile una alta persistencia en el sistema bancario teniendo en cuenta que en ambos bloques de tiempo analizados permanecen en un estado bancario y con una alta persistencia de 0.94 y 0.82 respectivamente, lo que se complementa con la tendencia constante al aumento del crédito bancario al sector privado en ambos estados. En el caso de México, existe una alta tendencia a la no permanencia en un sistema financiero bancario en el caso de generarse una propensión al aumento del crédito interno al sector privado. Esto se observa en la disminución en 0.29 de la probabilidad de permanencia del primer al segundo estado como se observa en la tabla 7. Para el caso de Brasil se observa un estricto cambio de estado, de mixto a bancario en el cual, según la figura 5 se genera por un aumento del nivel de crédito al sector privado en un $30 \%$, sin embargo mantiene una baja persistencia de permanencia en ese estado bancario en un segundo estado, siendo tan solo del 0.67 según la columna 7 .

En el análisis de los resultados para el segundo bloque de países se observa una fuerte tendencia a la adopción de un sistema financiero bursátil en un primer estado para el caso de Japón, que muestra una persistencia en el estado 1 de (0.83), además de una disminución del $80 \%$ en el nivel de crédito bancario al sector privado como sugiere la figura 5. Para el caso de Reino Unido se observa un fuerte aumento sobre el nivel de crédito bancario al sector privado, tocando su punto más alto en el año 2009, pasando de $120 \%$ a $180 \%$ sobre el porcentaje del PIB en nueve años. Sin embargo, se denota una "incomodidad" de permanencia en el mismo, teniendo en cuenta que la probabilidad de permanencia es baja (0.68) y que para el segundo momento registra una fuerte adopción de un sistema financiero bursátil, teniendo una disminución del $70 \%$ del nivel de crédito bancario, retomando los mismos niveles del año 2000.

Un camino distinto sigue Estados Unidos, país que a lo largo del periodo de evaluación ha tenido el nivel más bajo de crédito bancario al sector privado entre los países evaluados, nivel del 50\% sobre el PIB. La adopción del sistema financiero bursátil ha sido constante y estricta en ambos estados. Finalmente, para el caso de Alemania se observa una permanencia constante y estricta en un sistema financiero bancario, teniendo una persistencia de 0.92 en el primer estado bancario y una de 0.61 en el segundo momento, durante el cual se observa una leve disminución del nivel de crédito bancario al sector privado-mixto. Estas estimaciones finalmente nos muestran que no se puede rechazar la hipótesis nula de relación entre las variables estudiadas y su impacto sobre la permanencia de un país en un sistema financiero bancario o bursátil.

\section{Conclusiones}

En este documento investigamos el impacto de las variables: 1 . Acciones negociadas como \% del PIB. 2. Crédito bancario al sector privado como porcentaje del PIB. 3. PIB per cápita y 4 . Inflación como agentes generadores de transición de estados definidos como la adopción de un sistema financiero bancario o bursátil en un grupo de ocho países, que se dividen en dos bloques ambos pertenecientes a la OCDE. Para esto, nos beneficiamos de información del Banco Mundial y el Fondo Monetario Internacional. De igual manera, proponemos un marco teórico que nos permitió identificar la prevalencia del tipo de sistema financiero en cada país y sus características principales. Se destacan tres conclusiones principales.

Primero, encontramos una permanencia estricta en el estado bancario a través del tiempo en los países Latinoamericanos, además de una persistencia en el primer estado 
del que parte el estudio la cual es en promedio del (0.90), en comparación del segundo estado, que es del $(0,75)$. Sin embargo, la alta diferencia (0.15) de media probabilística permite entender que este bloque de países tiene un bajo nivel de adopción al cambio de sistema, esto es congruente con la coyuntura variable que caracteriza a este tipo de economías las cuales se han ido moldeando para contrarrestar los efectos focales generados por diferentes crisis y bonanzas.

Segundo, los resultados sugieren para el segundo bloque de países una leve tendencia a permanecer en el primer estado del que se parte al comienzo del periodo de estudio, con una diferencia de media probabilística de permanencia entre el primer $(0,78)$ y segundo $(0,73)$ estado, que es de $(0,05)$. De igual manera, se observa una alta variabilidad entre estados en los casos de Reino Unido y Japón, los cuales adoptan nuevos sistemas financieros hasta el año 2008. La buena estructuración de estas economías les ha permitido afrontar crisis, logrando contrarrestar sus efectos secundarios y dándoles la oportunidad en algún periodo de tiempo que abarca este trabajo, a algunos países, como Japón, de adoptar esporádicamente un sistema financiero mixto o fortalecerlos como Estados Unidos.

Finalmente, se recalca el alto nivel de significancia del nivel de crédito bancario al sector privado y el valor inflacionario en los diferentes estados de análisis en los países de ambos bloques. Estas variables, asociadas al impacto sobre el desarrollo productivo nacional resultan ser un agente disuasivo para la adopción de un sistema financiero especifico, dejando entrever la relación recíproca entre la construcción de un completo aparato producto nacional y el crecimiento económico de acuerdo al enfoque de la política financiera que desarrolle cada país. Dado que los sistemas financieros bancarios se asocian con empresas de tipo industrial y agrícola, mientras que los sistemas bursátiles se asocian con empresas del sector servicios, la innovación y la diversificación del riesgo por parte de los agentes, estos resultados sugie- ren que los países latinos pertenecientes a la OCDE no generarían una transición hacia este tipo de industrias autónomamente dada su persistencia a los sistemas bancarios. Los tomadores de decisiones en estos países deberían proponer políticas que encausarán a dichos países por un uso más apropiado de los mercados bursátiles como una forma de promover la industria de alta tecnología y los mercados de diversificación de riesgo necesarios para soportar dichas iniciativas.

\section{REFERENCIAS}

Aghion, P., Howitt, P. \& Mayer-Foulkes, D. (2005). TheEffectofFinancialDevelopment on Convergence: Theory and Evidence. The Quarterly Journal of Economics, 120(1). 173-222. http://dx.doi. org/10.1162/0033553053327515

Allen, F., Bartiloro, L., Gu, X. \& Kowalewski, O. (2018). Does economic structure determine financial structure? Journal of International Economics, 114(1). 389-409. http://dx.doi. org/10.1016/J.JINTECO.2018.08.004

Allen, F. \& Gale, D. (2000). Comparing financial systems. Cambridge: MIT Press.

Allen, F., Gu, X. \& Kowalewski, O. (2017). Financial Structure, Economic Growth and Development. [IÉSEG Working Paper Series 2017-ACF-04]. Recuperado de https://papers.ssrn.com/sol3/papers.cfm?abstract_id $=3035298$

Beck, T. \& Levine, R. (2002). Industry growth and capital allocation:: does having a market- or bank-based system matter? Journal of Financial Economics, 64(2). 147-180. http://dx.doi. org/10.1016/S0304-405X(02)00074-0

Betancourt, R., Misas, M. y Bonilla, L. (2011). La transmisión de las tasas de interés en Colombia: un enfoque multivariado con cambio de régimen [Presentación]. Recuperado de http://www. banrep.gov.co/sites/default/files/publicaciones/archivos/mec4.pdf 
Bruno, G., De Bonis, R. \& Silvestrini, A. (2012). Do financial systems converge? New evidence from financial assets in OECD countries. Journal of Comparative Economics, 40(1). 141-155. http:// dx.doi.org/10.1016/J.JCE.2011.09.003

Calvo, A. Parejo, J., Rodríguez, L. y Cuervo, Á. (2014). Manual del sistema financiero español (25 ed.). Barcelona: Ariel Economía y Empresa.

Čihák, M., Demirgüç-Kunt, A., Feyen, E. \& Levine, R. (2012). Benchmarking Financial Systems Around the World. [Working Paper 6175]. Washington, D.C.: World Bank. http:// documents.worldbank.org/curated/en/868131468326381955/pdf/ wps6175.pdf

Demirgüç-Kunt, A. \& Levine, R. (2001). Financial structure and economic growth: a cross-country comparison of banks, markets, and development. Cambridge: MIT Press.

Dong, K., Hong, S. \& Myung, H. (2018). 'A study on Classification of Insider threat using Markov Chain Model', KSII Transactions on Internet and Information Systems, 12(4). http://dx.doi. org/10.3837/tiis.2018.04.027.

Ergungor, O. (2004). Market- vs. bankbased financial systems: Do rights and regulations really matter? Journal of Banking \& Finance, 28(12). 2869$2887 . \quad$ http://dx.doi.org/10.1016/J. JBANKFIN.2003.04.001

Freixas, X. \& Rochet, J-C. (2008). Microeconomics of banking. Cambridge: MIT Press.

Gerschenkron, A. (1962). Economic backwardness in historical perspective : a book of essays. Cambridge: Harvard University Press.

Goutte, S. (2014). Conditional Markov regime switching model applied to economic modelling. Economic Modelling, 38(c), 258-269. http://dx.doi. org/10.1016/j.econmod.2013.12.007
Herger, N., Hodler, R. \& Lobsiger, M. (2008). What Determines Financial Development? Culture, Institutions or Trade', Review of World Economics, 144(3). 558-587. http://dx.doi. org/10.1007/s10290-008-0160-1

IMF, IFS and data files, and World Bank and OECD GDP estimates. (julio 10, 2018). Domestic credit to private sector by banks (\% of GDP). [Fichero de datos]. Recuperado de https://data. worldbank.org/indicator/FS.AST. PRVT.GD.ZS

Kaufmann, S. \& Valderrama, M. (2008). Bank lending in Germany and the UK: are there differences between a bank-based and a market-based country? International Journal of Finance \& Economics, 13(3). 266-279. http://dx.doi.org/10.1002/ijfe.345

Lee, B. (2012). Bank-based and marketbased financial systems: Timeseries evidence. Pacific-Basin Finance Journal, 20(2). 173-197. http://dx.doi.org/10.1016/J.PACFIN.2011.07.006

Levine, R. (2002). Bank-Based or MarketBased Financial Systems: Which is Better? [Working Paper 9138]. http:// dx.doi.org/10.3386/w9138

Levine, R. (1997). Financial Development and Economic Growth: Views and Agenda. Journal of Economic Literature, 35(2). 688-726. Recuperado de https://www.jstor.org/ stable/2729790

Levine, R. \& Zervos, S. (1999). Stock Markets, Banks, and Economic Growth. The World Bank (Policy Research Working Papers). http://dx.doi. org/10.1596/1813-9450-1690

Levine, R., Beck, T. \& Maksimovic, V. (2000). Financial Structure and Economic Development: Firm, Industry, and Country Evidence. [WPS2423]. http://dx.doi.org/10.1596/1813-94502423 
López, E. y Joa, L. (2001). Markov chains applied to the analyzis of the progress of research projets. Revista cubana de informática médica, 9(1). 44-51. Recuperado de http://www. medigraphic.com/cgi-bin/new/resumenI.cgi?IDARTICULO=72043

Luintel, K., Khan., M., Arestis, P. \& Theodoriditis, K. (2008). Financial structure and economic growth. Journal of Development Economics, 86(1). 181-200. http://dx.doi.org/10.1016/J. JDEVECO.2007.11.006

Merton, R. (1995). A Functional Perspective of Financial Intermediation. $\mathrm{Fi}$ nancial Management, 24(2). 23-41. http://dx.doi.org/10.2307/3665532

Prochniak, M. \& Wasiak, K. (2017). The impact of the financial system on economic growth in the context of the global crisis: empirical evidence for the EU and OECD countries. Empirica, 44(2). 295-337. http:// dx.doi.org/10.1007/s10663-0169323-9

Pussetto, L. (2008). Sistema financiero y crecimiento económico: Un misterio sin resolver. Palermo Business Review, 1(1). 47-60. Disponible en https://www.palermo.edu/economicas/cbrs/pdf/1Business04.pdf

Rajan, R. \& Zingales, L. (1998). Financial Dependence and Growth. [Working Paper 5758]. http://dx.doi. org/10.3386/w5758

Rajan, R. \& Zingales, L. (2003). Banks and Markets: The Changing Character of European Finance. [CRSP Working Paper No. 546]. http:// dx.doi.org/10.2139/ssrn.389100

Russ, K. \& Valderrama, D. (2012). A theory of bank versus bond finance and intra-industry reallocation. Journal of Macroeconomics, 34(3). 652-673. http://dx.doi.org/10.1016/J.JMACRO.2012.05.004
Stulz, R. (2000). Financial Structure, Corporate Finance and Economic Growth. International Review of Finance, 1(1). 11-38. http://dx.doi. org/10.1111/1468-2443.00003

Terceño, A. y Guercio, B. (2011). El crecimiento económico y el desarrollo del sistema financiero. Un análisis comparativo. Investigaciones Europeas de Dirección y Economía de la Empresa. 17(2). 33-46. http://dx.doi. org/10.1016/S1135-2523(12)60051-3

Zogning, F. (2017). Comparing financial systems around the world: capital markets, legal systems, and governance regimes. Economics, Management, and Financial Markets, 12(4). 43-58. http://dx.doi. org/10.22381/EMFM12420172

\section{Biodata}

Christian Camilo Anzola Morales Magister en Economía de la Universidad del Rosario. Economista de la Universidad Militar Nueva Granada. Asistente de Investigación en la Universidad Militar Nueva Granada. https://orcid.org/00000003-1375-2597

Paola Sophia Vargas Pachón Economista de la Universidad Militar Nueva Granada. Asistente de Investigación en la Universidad Militar Nueva Granada. https://orcid.org/0000-0002-6386-4557

\footnotetext{
Alberto Méndez Morales Ph.D. y Máster en Economía y Gestión de la Innovación de la Universidad Autónoma de Madrid, especialista en evaluación económica y social de proyectos de la Universidad de los Andes, Economista de la Universidad Externado de Colombia. Docente de tiempo completo de la Universidad Militar Nueva Granada. https://orcid.org/0000-00017971-5305
} 


\section{Anexos}

TABLA 8

Resultados MSDR para México

\begin{tabular}{|c|c|c|c|c|c|}
\hline Variables & Estado 1 & Estado 2 & lnsigma & $\mathrm{p} 11$ & $\mathrm{p} 21$ \\
\hline \multirow[t]{3}{*}{ Cred_PIB } & $0.407^{* * *}$ & $0.591^{* * *}$ & & & \\
\hline & $(0)^{1}$ & $(0)^{1}$ & & & \\
\hline & $(0.030)^{2}$ & $(0.044)^{2}$ & & & \\
\hline \multirow[t]{3}{*}{ PIB_perc } & $0.959^{* * *}$ & 0.129 & & & \\
\hline & $(0)^{1}$ & $(0.309)^{1}$ & & & \\
\hline & $(0.223)^{2}$ & $(0.126)^{2}$ & & & \\
\hline \multirow[t]{3}{*}{ Inflac } & $-0.267^{* *}$ & -0.207 & & & \\
\hline & $(0.0160)^{1}$ & $(0.111)^{1}$ & & & \\
\hline & $(0.110)^{2}$ & $(0.129)^{2}$ & & & \\
\hline \multirow[t]{2}{*}{ Constante } & & & 0.855 & 0.679 & 0.426 \\
\hline & & & $(0.171)^{2}$ & $(0.174)^{2}$ & $(0.232)^{2}$ \\
\hline $\mathrm{N}$ & 17 & 17 & 17 & 17 & 17 \\
\hline
\end{tabular}

P-Value en paréntesis 1. Errores estándar en paréntesis $2 .{ }^{* * *} \mathrm{p}<0.01{ }^{* *} \mathrm{p}<0.05{ }^{*} \mathrm{p}<0.1$

Fuente: Banco Mundial 2000-2016. Cálculos propios.

TABLA 9

Resultados MSDR para Colombia

\begin{tabular}{|c|c|c|c|c|c|}
\hline Variables & Estado 1 & Estado 2 & lnsigma & p11 & $\mathrm{p} 21$ \\
\hline \multirow[t]{3}{*}{ Cred_PIB } & -0.0483 & $0.182^{* * *}$ & & & \\
\hline & $(0.869)^{1}$ & $(0.000422)^{1}$ & & & \\
\hline & $(0.292)^{2}$ & $\left(0.051^{22}\right.$ & & & \\
\hline \multirow[t]{3}{*}{ PIB_perc } & 18.54 & $42.67^{*}$ & & & \\
\hline & $(0.733)^{1}$ & $(0.0834)^{1}$ & & & \\
\hline & $(54.343)^{2}$ & $(24.641)^{2}$ & & & \\
\hline \multirow[t]{3}{*}{ Inflac } & 0.337 & 0.107 & & & \\
\hline & $(0.807)^{1}$ & $(0.701)^{1}$ & & & \\
\hline & $(1.378)^{2}$ & $(0.278)^{2}$ & & & \\
\hline \multirow[t]{2}{*}{ Constante } & & & 1.395 & 0.895 & 0.055 \\
\hline & & & $(0.247)^{2}$ & $(0.139)^{2}$ & $(0.061)^{2}$ \\
\hline $\mathrm{N}$ & 16 & 16 & 16 & 16 & 16 \\
\hline
\end{tabular}

P-Value en paréntesis 1. Errores estándar en paréntesis $2 .{ }^{* * *} \mathrm{p}<0.01{ }^{* *} \mathrm{p}<0.05{ }^{*} \mathrm{p}<0.1$

Fuente: Banco Mundial 2000-2016. Cálculos propios. 
TABLA 10

Resultados MSDR para Brasil

\begin{tabular}{|c|c|c|c|c|c|}
\hline Variables & Estado 1 & Estado 2 & lnsigma & $\mathrm{p} 11$ & $\mathrm{p} 21$ \\
\hline \multirow[t]{3}{*}{ Cred_PIB } & $0.446^{* * *}$ & $0.791^{* * *}$ & & & \\
\hline & $(0)^{1}$ & $(0)^{1}$ & & & \\
\hline & $(0.027)^{2}$ & $(0.068)^{2}$ & & & \\
\hline \multirow[t]{3}{*}{ PIB_perc } & $136.22^{*}$ & 30.22 & & & \\
\hline & $(0.075)^{1}$ & $(0.818)^{1}$ & & & \\
\hline & $(76.435)^{2}$ & $(131.08)^{2}$ & & & \\
\hline \multirow[t]{2}{*}{ Constante } & & & 4.14 & 0.918 & 0.332 \\
\hline & & & $(0.813)^{2}$ & $(0.081)^{2}$ & $(0.306)^{2}$ \\
\hline $\mathrm{N}$ & 15 & 15 & 15 & 15 & 15 \\
\hline \multicolumn{6}{|c|}{$\begin{array}{l}\text { i. El desarrollo del modelo final para el caso Brasil implicó la selección de dos variables independientes } \\
\text { al contrario de los demás países que ejecutaron tres. Esta acción se llevó a cabo teniendo en cuenta que } \\
\text { la variable inflación no resultó ser significativa y demostró efectos de auto correlación serial de primer } \\
\text { orden entre los residuales. }\end{array}$} \\
\hline
\end{tabular}

P-Value en paréntesis 1. Errores estándar en paréntesis $2 .{ }^{* * *} \mathrm{p}<0.01^{* *} \mathrm{p}<0.05^{*} \mathrm{p}<0.1$

Fuente: Banco Mundial 2000-2016. Cálculos propios.

TABLA 11

Resultados MSDR para Chile

\begin{tabular}{|c|c|c|c|c|c|}
\hline Variables & Estado 1 & Estado 2 & Lnsigma & p11 & $\mathrm{p} 21$ \\
\hline \multirow[t]{3}{*}{ Cred_PIB } & 0.028 & $0.202^{* * * *}$ & & & \\
\hline & $(0.160)^{1}$ & $(0)^{1}$ & & & \\
\hline & $(0.019)^{2}$ & $(0.019)^{2}$ & & & \\
\hline \multirow[t]{3}{*}{ PIB_perc } & 0.137 & 0.110 & & & \\
\hline & $(0.784)^{1}$ & $(0.719)^{1}$ & & & \\
\hline & $(0.500)^{2}$ & $(0.303)^{2}$ & & & \\
\hline \multirow[t]{5}{*}{ Inflac } & $1.390^{* * *}$ & $1.588^{* * *}$ & & & \\
\hline & $(0)^{1}$ & $(0)^{1}$ & & & \\
\hline & $(0.352)^{2}$ & $(0.252)^{2}$ & & & \\
\hline & & $\begin{array}{r}\text { Cons } \\
1.9 \\
0.9 \\
0.1\end{array}$ & & & \\
\hline & & & $(0.335)^{2}$ & $(0.082)^{2}$ & $(0.134)^{2}$ \\
\hline $\mathrm{N}$ & 17 & 17 & 17 & 17 & 17 \\
\hline
\end{tabular}

P-Value en paréntesis 1. Errores estándar en paréntesis $2 .{ }^{* * *} \mathrm{p}<0.01^{* *} \mathrm{p}<0.05^{*} \mathrm{p}<0.1$

Fuente: Banco Mundial 2000-2016. Cálculos propios. 
TABLA 12

Resultados MSDR para Alemania

\begin{tabular}{|c|c|c|c|c|c|}
\hline Variables & Estado 1 & Estado 2 & lnsigma & p11 & $\mathrm{p} 21$ \\
\hline \multirow[t]{3}{*}{ Cred_PIB } & $0.450^{* * * *}$ & $1.381^{* * * *}$ & & & \\
\hline & $(0)^{1}$ & $(0)^{1}$ & & & \\
\hline & $(0.048)^{2}$ & $(0.134)^{2}$ & & & \\
\hline \multirow[t]{3}{*}{ PIB_perc } & $2.319^{* *}$ & $4.823^{* * *}$ & & & \\
\hline & $(0.017)^{1}$ & $(0)^{1}$ & & & \\
\hline & $(0.968)^{2}$ & $(1.002)^{2}$ & & & \\
\hline \multirow[t]{3}{*}{ Inflac } & -1.792 & $-32.69^{* * *}$ & & & \\
\hline & $(0.564)^{1}$ & $(0.000175)^{1}$ & & & \\
\hline & $(3.107)^{2}$ & $(8.711)^{2}$ & & & \\
\hline \multirow[t]{2}{*}{ Constante } & & & 6.210 & 0.921 & 0.387 \\
\hline & & & $(1.111)^{2}$ & $(0.079)^{2}$ & $(0.270)^{2}$ \\
\hline $\mathrm{N}$ & 16 & 16 & 16 & 16 & 16 \\
\hline
\end{tabular}

P-Value en paréntesis 1. Errores estándar en paréntesis $2 .{ }^{* * *} \mathrm{p}<0.01^{* *} \mathrm{p}<0.05^{*} \mathrm{p}<0.1$ Fuente: Banco Mundial 2000-2016. Cálculos propios.

TABLA 13

Resultados MSDR para Japón

\begin{tabular}{|c|c|c|c|c|c|}
\hline Variables & Estado 1 & Estado 2 & lnsigma & p11 & $\mathrm{p} 21$ \\
\hline \multirow[t]{3}{*}{ Constante } & $60.04^{* * *}$ & $117.5^{* * *}$ & 15.005 & 0.825 & 0.205 \\
\hline & $(0)^{1}$ & $(0)^{1}$ & $(2.69)^{2}$ & $(0.121)^{2}$ & $(0.144)^{2}$ \\
\hline & $(5.282)^{2}$ & $(5.880)^{2}$ & & & \\
\hline $\mathrm{N}$ & 17 & 17 & 17 & 17 & 17 \\
\hline
\end{tabular}

P-Value en paréntesis 1. Errores estándar en paréntesis $2 .{ }^{* * *} \mathrm{p}<0.01{ }^{* *} \mathrm{p}<0.05{ }^{*} \mathrm{p}<0.1$

Fuente: Banco Mundial 2000-2016. Cálculos propios. 
TABLA 14

Resultados MSDR para Reino Unido

\begin{tabular}{llllll}
\hline \multicolumn{1}{c}{ Variables } & \multicolumn{1}{c}{ Estado 1} & \multicolumn{1}{c}{ Estado 2} & lnsigma & p11 & p21 \\
Cred_PIB & $0.628^{* * * *}$ & $0.749^{* * *}$ & & & \\
& $(0)^{1}$ & $(0)^{1}$ & & & \\
& $(0.067)^{2}$ & $(0.047)^{2}$ & & & \\
PIB_perc & -1.031 & $15.21^{* * *}$ & & & \\
$(0.449)^{1}$ & & & & & \\
$(0)^{1}$ & & & & & \\
& $(1.361)^{2}$ & $(1.714)^{2}$ & & & \\
Inflac & -7.982 & $-11.61^{* * *}$ & & & \\
& $(0.129)^{1}$ & $(0.001)^{1}$ & & & \\
& $(5.264)^{2}$ & $(3.535)^{2}$ & & & \\
Constante & & & 5.835 & 0.680 & $(1.070)^{2}$ \\
& & & 15 & $(0.160)^{2}$ & $(0.198)^{2}$ \\
\hline $\mathrm{N}$ & 15 & 15 & & 15 & 15 \\
\hline
\end{tabular}

P-Value en paréntesis 1. Errores estándar en paréntesis $2 .{ }^{* * *} \mathrm{p}<0.01^{* *} \mathrm{p}<0.05^{*} \mathrm{p}<0.1$

Fuente: Banco Mundial 2000-2016. Cálculos propios.

TABLA 15

Resultados MSDR para Estados Unidos

\begin{tabular}{|c|c|c|c|c|c|}
\hline Variables & Estado 1 & Estado 2 & lnsigma & $\mathrm{p} 11$ & $\mathrm{p} 21$ \\
\hline \multirow[t]{3}{*}{ Constante } & $169.3^{* * *}$ & $243.3^{* * *}$ & 35.769 & 0.706 & 0.089 \\
\hline & & $(0)^{1}$ & $(6.784)^{2}$ & $(0.220)^{2}$ & $(0.100)^{2}$ \\
\hline & $(22.994)^{2}$ & $(11.851)^{2}$ & & & \\
\hline $\mathrm{N}$ & 17 & 17 & 17 & 17 & 17 \\
\hline $\begin{array}{l}\text { iii. Para el caso de Estados Unidos, no se } \\
\text { observaron resultados congruentes en el MSDR } \\
\text { con todas las variables definidas, para lo cual } \\
\text { se realiza un ejercicio adicional en donde se } \\
\text { modela un MSDR con una sola variable (Crédito } \\
\text { bancario al sector privado como porcentaje del } \\
\text { PIB) para dos estados, la cual termina siendo } \\
\text { significativa y dando como resultado mayor } \\
\text { bondad de ajuste al modelo final. }\end{array}$ & & & & & \\
\hline
\end{tabular}

P-Value en paréntesis 1. Errores estándar en paréntesis 2. ${ }^{* * *} \mathrm{p}<0.01^{* *} \mathrm{p}<0.05^{*} \mathrm{p}<0.1$

Fuente: Banco Mundial 2000-2016. Cálculos propios. 